\title{
Effects of delayed Kerr nonlinearity and ionization on the filamentary ultrashort laser pulses in air
}

\author{
M. Nurhuda ${ }^{1,2}$ and E. van Groesen ${ }^{2}$ \\ ${ }^{1}$ Physics Department, Brawijaya University, Malang, 65144, Indonesia \\ ${ }^{2}$ Department of Applied Mathematics, University of Twente, 7500 AE Enschede, The Netherlands \\ (Received 14 July 2004; revised manuscript received 3 January 2005; published 24 June 2005)
}

\begin{abstract}
We present a systematic study of filamentary ultrashort laser pulses in air, through numerical solutions of the nonlinear Schrödinger equation for various contributions of the delayed Kerr nonlinearity. The results show that a relatively larger contribution of the delayed Kerr nonlinearity will lead to a longer stable filament. This is explained from the transfer of the nonlinear contributions from the frontier part to the back of the pulse and the counterbalanced action of the negative plasma induced nonlinearity by the delayed Kerr nonlinearity in the trailing part of the pulses. Furthermore, effect of ionization on the stability of the filament is investigated. Two formulas are used to generate the data of the ionization, i.e., the Perelemov, Popov, and Terent'ev (PPT) and the Ammosov, Delone, and Krainov formula. It is found that simulation with higher ionization rate (PPT) could generate a more stable and longer filament.
\end{abstract}

DOI: 10.1103/PhysRevE.71.066502

PACS number(s): 52.50.Jm, 42.65.Jx, 42.65.Sf

\section{INTRODUCTION}

Recent progress in laser technology has led to the generation of a table top Terra watt laser pulse with a pulse duration of less than 100 fs (see, e.g., Refs. [1,2]). Numerous experiments have been carried out using such ultrashort laser pulses for a wide range of purposes, either for scientific or for technological applications [3-5]. In particular, the nonlinear dynamics of ultrashort, intense laser pulse in transparent media, e.g., fused silica, air molecules or gases, have attracted considerable interest over the last decades because of the apparent difference in behavior from that of long or continuous wave $(\mathrm{cw})$ laser. The interaction of such intense, ultrashort laser fields with matter provides, in addition to the well-known self-focusing phenomenon, a wealth of unexpected interesting phenomena, e.g., pulse splitting, pulse steepening, plasma defocusing, self-guided beam propagation, and white light continuum generation (see, e.g., Refs. [6-10]). Related to self-focusing, for instance, the high intensity laser field strongly induces the nonlinear refractive index, and drives the beam to refract from a domain of lower intensity to one of higher intensity. The intensity may become several times higher than that of the initial field, such that it could ionize air molecules. Experiments using ultrashort $(\approx 100 \mathrm{fs})$, high intensity $\left(I>10^{13} \mathrm{~W} / \mathrm{cm}^{2}\right)$ laser pulses have demonstrated long-distance self-guided atmospheric propagation, filamentation, and white light generation [11]. In addition, self-phase modulation, stimulated Raman scattering, and plasma formation also contribute to a considerable spectral broadening and white light generation [12].

To the best of our knowledge, the mechanism of pulse propagation over extended distances should involve a combination of a moving focus model for the initial stage of propagation [7], followed by modulation instability due to power replenishment $[9,13]$ and then followed by a selfguiding mechanism (see, e.g., Refs. $[14,15]$ ). The moving focus model predicts the nonlinear focal length that depends on the laser power through the optical Kerr effect, and extended propagation is formed through self-focusing of the beam in the different time slices [7]. In the next stage of propagation, refocusing takes place repeatedly in the trailing edge through the replenishment of power from the peripheral area [9]. During this stage, multiple pulse splitting and transverse ring formation of the intensity profile occur (see, e.g., Refs. $[9,12,13])$. The multiphoton ionization (MPI) limits the growth of intensity and the pulse splits further in parts of much smaller duration, each of which contains power that is slightly greater than the critical power for self-focusing $P_{\text {cr }}$ $=1.99 \lambda^{2} / 4 \pi n_{2}$, where $\lambda$ is the wavelength and $n_{2}$ the nonlinear refractive index. The generated tiny plasma elements in each splitting then guide the pulse in the rest of propagation, through self-guiding mechanism due to a quasibalance between Kerr nonlinearity, diffraction, and plasma induced negative nonlinearity. As a result, the pulse can propagate further over distances that are several tens longer than the corresponding diffraction length, and will finally diffract when the power maintaining the balances decreases to below the critical power. Recently, Ref. [16], for the first time an analytical result was described that predicts the length of the filament in terms of energy, power, and binding energy of the atomic or molecular constituents of the medium, whose result is, in principle, valid if the power is approximately (but slightly greater) than the critical power for self-focusing. However, if the laser power is several times larger than the critical power, the mechanism near the focal point is still inherently complex and needs full simulation of the extended nonlinear Schrödinger equation.

While the mechanism of pulse propagation in atmosphere is well understood for long pulses, this is not the case for sub-100 femtosecond laser pulses. The physics governing the propagation of short intense laser pulses in air can be very different from that of long laser pulses, due to, for instance, the delayed Kerr nonlinearity associated with the molecular rotational modes or Raman transition, self-steepening, group velocity dispersion, and spatiotemporal self-focusing (see, 


$$
\mathrm{g}=1, \quad \mathrm{~g}^{*}=0
$$

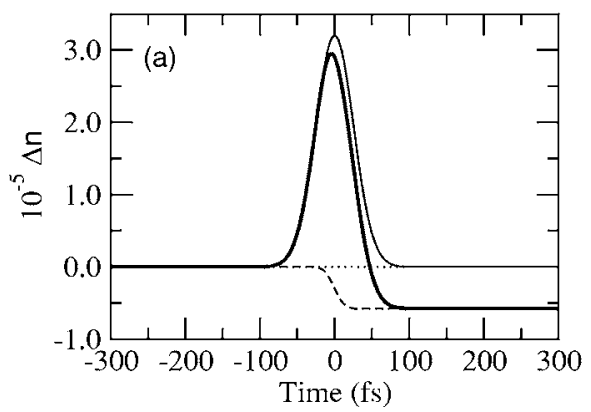

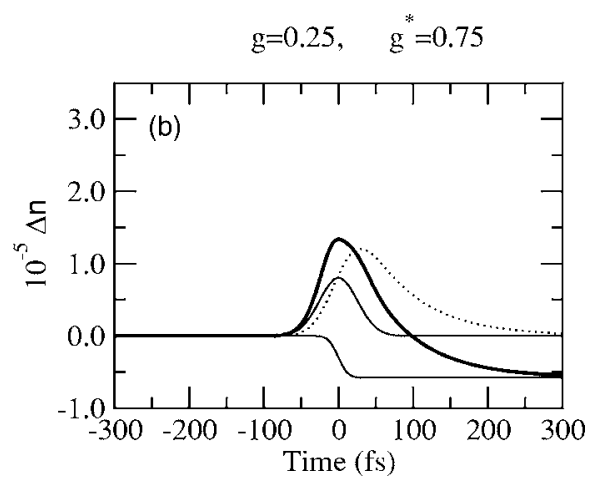

FIG. 1. Plots showing the individual contributions to the nonlinear refractive index for two different pairs of $\left(g, g^{*}\right)$; thin lines account for purely Kerr effect, dotted curves for delayed Kerr effect, dashed curves for contributions by plasma, and the thick lines stand for sum of them. e.g., Ref. [17]). The effective nonlinear refractive index of air, which is effectively constant and instantaneous in the case of long pulse propagation, will become time dependent if the pulse duration is shorter than the characteristic period associated with the stimulated Raman transition of the atomic or molecular medium. This implies that the nonlinear refractive index of air could be a function of the laser pulse length. Consequently, sub-100 femtosecond laser pulses will experience smaller effective nonlinear refractive index changes than pulses of a picosecond or even nanosecond [16]. In addition, higher order (than third order) nonlinear susceptibility has also to be taken into account if the intensity goes beyond the value where the perturbation theory breaks down [18].

In most simulation models of femtosecond pulse propagation in air the Kerr nonlinearity is usually assumed to be instantaneous (e.g., Refs. [19,20]) or consists of the delayed part with the same fraction of the instantaneous part (see, e.g., Refs. [9,14,21]). The latter was based on experimental results of spectral broadening of an unfocused 125 femtosecond pulse in air [22,23]. However, it remains unclear, whether the ratio from delayed to the instantaneous part remains the same or becomes smaller or larger if the pulse lengths are made shorter, e.g., 60 fs or even shorter. This is because the delayed Kerr nonlinearity originates from the inertial molecular dynamics (e.g., vibration and rotation), whose delay time is in the order of 100 femtosecond (or less), while the instantaneous Kerr nonlinearity arises from the electronic response of the electrons orbiting around the nucleus. Unfortunately, there is neither analytical prediction nor numerical result that can be used to determine the ratio from delayed to the instantaneous, even for long pulses. Since this ratio is still in question, it seems interesting to systematically investigate the dynamics of the propagation of ultrashort for given various fraction of the delayed nonlinearity. The delayed part is of particular interest, since its presence could reduce the effect of self-focusing effect in front of the laser pulses, while it could compensate the negative induced-plasma refractive index that is exerted in the trailing edge of the pulses and hence strongly modifies the dynamics of propagation, such that it may significantly stabilize the pulse propagation.

Furthermore, in most simulations of femtosecond propagation in air, the ionization rate is usually generated by using Keldysh's formula [24] for an intensity regime where the multiphoton ionization is assumed to be dominant (low in- tensity) and by using Ammosov, Delone, Krainov (ADK) formula [25] for high intensity or tunneling regime. However, it has been shown that the ionization rate for air molecules derived from ADK formula is much lower than the experimental data, particularly for intensity smaller than 2 $\times 10^{14} \mathrm{~W} / \mathrm{cm}^{2}$ [26]. A best fit ionization rate data could be reproduced if a semianalytic, the Perelemov, Popov, and Terent'ev (PPT) formula with an appropriate effective nuclear charge is used. To which extent a discrepancy in the ionization rate modifies the dynamics of the filaments is therefore interesting here to study.

In this paper, we present simulation results of femtosecond pulse propagation in air contributed by various fraction of the delayed nonlinearity. The results show that higher contribution of delayed nonlinearity could lead into a more stable filament propagation. Effects of ionization on the stability of filament are also studied here.

The paper is organized as follows. In Sec. II we briefly review the theory underlying the simulation model. The simulation results will be presented and discussed in Sec. III. Finally, conclusions of our work will be presented in Sec. IV.

\section{THE MATHEMATICAL-PHYSICAL MODEL}

We start with a brief discussion of the model to be used for subsequent simulations. The model will incorporate the effect of spatiotemporal transverse diffraction, dispersion, instantaneous, and delayed Kerr nonlinearity, plasma effect, ionization, and energy depletion. We assume that the propagation of the pulses takes place along the $z$ axis, that there is rotational symmetry, and that the field is linearly polarized. Consider the electric field of the form

$$
E(r, z, t)=\frac{1}{2} \mathcal{E}(r, z, t) e^{-i \omega_{0} t+i k_{0} z}+\mathrm{c} . \mathrm{c} .,
$$

where $\mathcal{E}(r, z, t)$ is the complex envelope of the field and c.c. denotes the complex conjugate of the previous term. Within the slowly varying envelope (SVE) approximation the equation for the electric field envelope $\mathcal{E}(r, z, t)$ in the moving frame can be written down as [17,27-29]:

$$
\begin{aligned}
\frac{\partial \mathcal{E}(r, z, t)}{\partial z}= & i \frac{\nabla_{\perp}^{2}}{2 k_{0}}\left(1+\frac{i}{\omega_{0}} \frac{\partial}{\partial t}\right)^{-1} \mathcal{E}(r, z, t)-\frac{i k_{0}^{\prime \prime}}{2} \frac{\partial^{2} \mathcal{E}(r, z, t)}{\partial t^{2}} \\
& +i k_{0}\left[\left(1+\frac{i}{\omega_{0}} \frac{\partial}{\partial t}\right) \Delta n_{K}(t)+\left(1-\frac{i}{\omega_{0}} \frac{\partial}{\partial t}\right)\right. \\
& \left.\times \Delta n_{P}(t)\right] \mathcal{E}(r, z, t)-\frac{\alpha}{2} \mathcal{E}(r, z, t),
\end{aligned}
$$



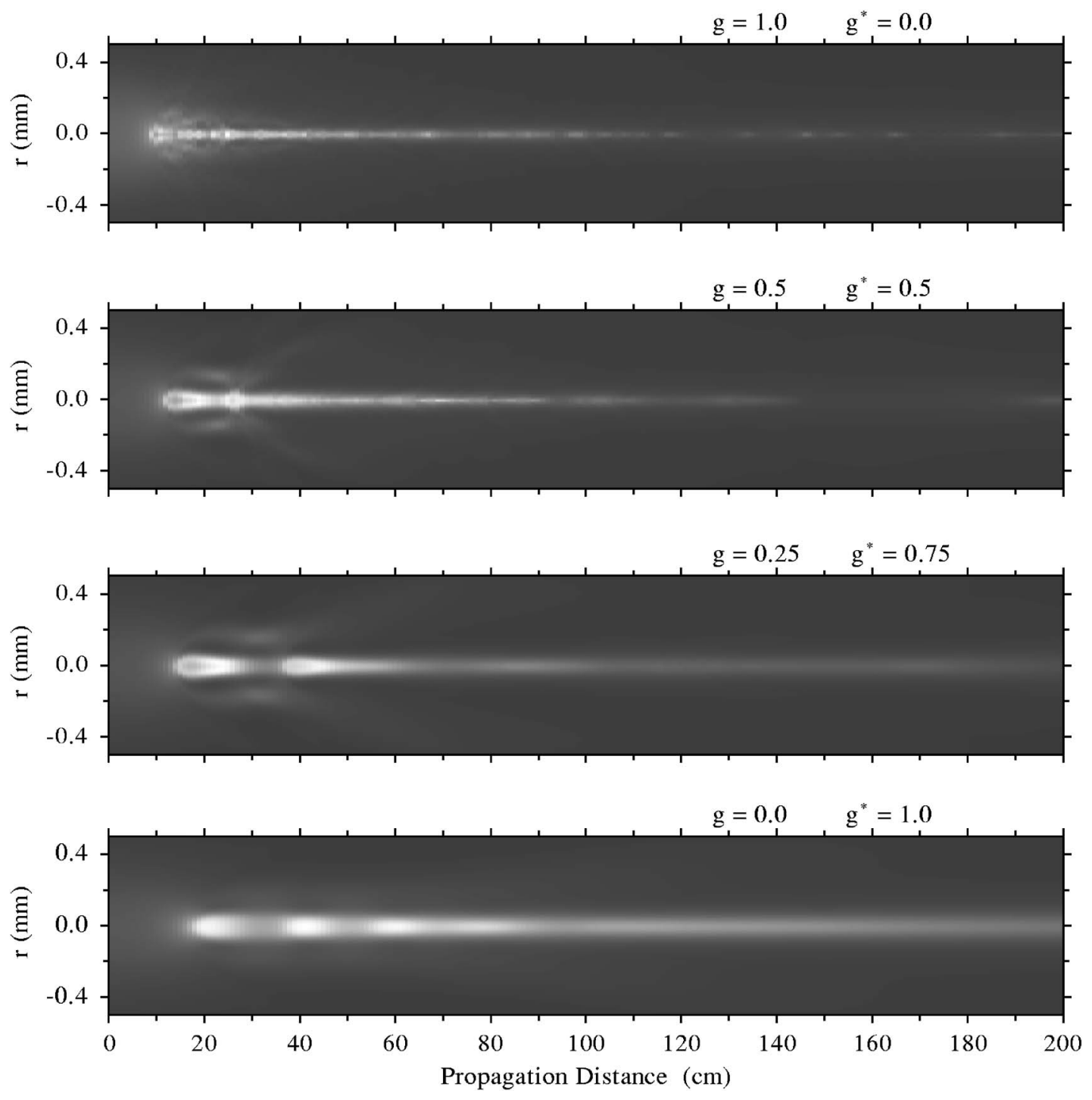

FIG. 2. The portraits of intensity profile $I_{\mathrm{av}}$ (calculated by averaging the instantaneous intensity over whole interval of pulse duration) as a function of propagation distance, obtained from the simulations using various pairs of $\left(g, g^{*}\right)$, i.e., $(1,0),(0.5,05),(0.25,0.75)$, and $(0,1)$, respectively, from top to bottom.

where the terms on the right-hand side account, respectively, for the beam diffraction, the group velocity dispersion (GVD), the refractive index change due to Kerr nonlinearity and plasma, and the absorption rate. The coefficient of the group velocity dispersion is taken to be $0.2 \mathrm{fs}^{2} / \mathrm{cm}$ [14]. The Kerr nonlinearity of air consists of two terms, i.e., the instantaneous electronic and the delayed part contributed by the stimulated Raman transition,

$$
\Delta n_{K}(t)=g n_{2} I(t)+g^{*} n_{2} \int_{-\infty}^{t} R\left(t-t^{\prime}\right) I\left(t^{\prime}\right) d t^{\prime},
$$

where $g$ and $g^{*}$ are parameters determining the contributions of the instantaneous and the delayed part, respectively. Here and in the following the intensity is defined as $I$ $=I_{A}|\mathcal{E}(r, z, t)|^{2}$ with $I_{A}=3.5 \times 10^{16} \mathrm{~W} / \mathrm{cm}^{2}$ is the atomic intensity. The response time function $R(t)$ has the form $[12,14]$

$$
R(t)=\frac{1}{\tau} e^{-t / \tau} \text { for } t>0,
$$

where $\tau$ is the delay time, fixed to be $70 \mathrm{fs}$ [23]. The nonlinear refractive index $n_{2}$ has a value of $6.4 \times 10^{-19} \mathrm{~cm}^{2} / \mathrm{W}$ [14], assuming that the atmosphere pressure is equal to unity. Another form of response time function can be used (see, e.g., Refs. $[9,30])$, but then the evaluation of the integral Eq. (3) will be much more time consuming.

The generation of plasma is expected to be mainly driven by the multiphoton ionization (MPI). The rate equation for the electron density $\rho$ reads

$$
\frac{\partial \rho(t)}{\partial t}=\Gamma(I)[1-\rho(t)]
$$

where $\Gamma(I)$ is the total ionization rate. For determining the ionization rate, we employed the PPT formula with an appropriate effective nuclear charge $Z_{\text {eff }}$ described elsewhere 

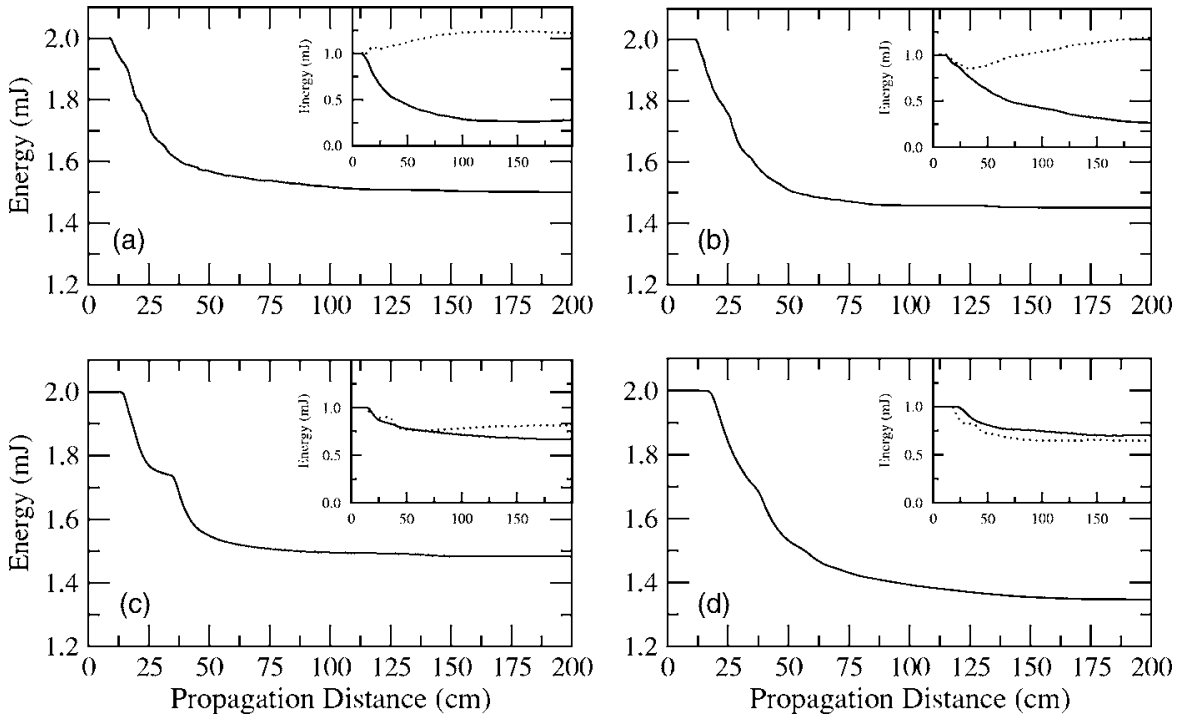

FIG. 3. Plot of total energy of the laser pulse for various pair of $\left(g, g^{*}\right)$ equal to $(1,0),(0.5,0.5)$, $(0.25,0.75)$, and $(0,1)$, respectively, for (a), (b), (c), and (d). In the insets the pulse energy in the leading part and in the trailing part is shown.
[26,31], which includes the Coulomb tail and has been proven to reproduce the ionization rate of air molecules correctly [26]. In addition, for the purpose of comparison of the stability of the filament with respect to the ionization rate being used, we also employed the ADK formula with the same value of $Z_{\text {eff. }}$. We did not include in the model the cascade ionization and electron recombination processes, since their contributions are small compared to that of MPI processes [14].

Since the principle constituents of air are $80 \%$ nitrogen and $20 \%$ oxygen molecules, the total electron density can be written as $\rho(t)=0.8 \rho^{N_{2}}(t)+0.2 \rho^{O_{2}}(t)$, where the superscripts indicate the individual contribution. The corresponding plasma refractive index has the form

$$
\Delta n_{P}(t)=-\frac{e^{2} \rho(t) N_{0}}{\epsilon_{0} m_{e} \omega_{0}^{2}},
$$

where $e, m_{e}$, and $\epsilon_{0}$ are the electron charge, electron mass, and vacuum permittivity, respectively, and where $N_{0}=2.688$ $\times 10^{19} \mathrm{~cm}^{-3}$ is the Loschmidt's number.

The rate of energy depletion $\alpha$ is related to the multiphoton absorption (MPA), given by $[27,32]$ :

$$
\alpha=\frac{N_{0} I_{A} \Gamma(I) l \hbar \omega}{I},
$$

where $l$ denotes the minimum number of photons required to ionize an electron in the ground state of energy $E_{b} \sim l \hbar \omega$. Alternatively, $\alpha$ can also be written as an imaginary part of the complex instantaneous nonlinearity [32]

$$
\alpha=\frac{k_{0} a_{0} e N_{0} \operatorname{Im}(\Delta \chi)}{\epsilon_{0} E_{0}},
$$

where $a_{0}$ and $E_{0}$ are the Bohr's radius of atomic hydrogen and atomic electric field $\left(\approx 5.1 \times 10^{11} \mathrm{~V} / \mathrm{m}\right)$ respectively.

The input electric field envelope is chosen to have both a spatial and a temporal Gaussian form and can then be written as

$$
\mathcal{E}(r, z, t)=\mathcal{E}_{0} \exp \left(-\frac{r^{2}}{r_{0}^{2}}-\frac{t^{2}}{\mathcal{T}^{2}}\right),
$$

where $\mathcal{E}_{0}=\sqrt{I_{0} / I_{A}}$ is the amplitude. The peak intensity $I_{0}$ can be written in the form of the pulse energy $\mathcal{U}$, the pulse width factor $\mathcal{T}$ the initial beam radius $r_{0}$,

$$
I_{0}=\sqrt{\frac{2}{\pi}} \frac{2 \mathcal{U}}{\mathcal{T} \pi r_{0}^{2}} .
$$

The pulse width factor $\mathcal{T}$ is related to the full width at halfmaximum (FWHM) by $\mathcal{T}=\sqrt{1 /(2 \log 2)} T_{\mathrm{FWHM}}$.

Equation (2) is solved using split-step Fourier-transform method, described elsewhere (see, e.g., Ref. [33] and references therein). The homogenous part is calculated in the frequency domain, and the nonlinear term is calculated in the time domain.

In the simulations, the maximum radial boundary $R_{\max }$ $=1.2 \mathrm{~cm}$ was discretized in a equidistance mesh size of $2 \mu \mathrm{m}$. The whole time interval was discretized in 512 points, while the propagation step $d z$ was set at a value of $0.1 \mathrm{~mm}$. The convergence test was performed by increasing the number of time steps $N$ from 512 to 1024, and by doubling the propagation step $d z$. We found that no significant change in the dynamics could be observed. Typical CPU time required was approximately $50 \mathrm{~h}$ on Intel Pentium IV/1800 Mhz processor for propagating the solution up to $2 \mathrm{~m}$.

\section{RESULTS AND DISCUSSION}

Figure 1 illustrates the contribution of the refractive index change due to instantaneous Kerr nonlinearity (thin solid lines), delayed Kerr nonlinearity (thin, dotted line), and plasma induced nonlinearity (dashed line) and the sum of the individual contributions (thick curve) for a given pair of $\left(g, g^{*}\right)=(1,0)$ and $\left(g, g^{*}\right)=(0.25,0.75)$.

The pulse is assumed to have Gaussian form with peak intensity equal to $5 \times 10^{13} \mathrm{~W} / \mathrm{cm}^{2}$. The instantaneous Kerr effect generates an index change $\Delta n$ which is simply proportional to intensity, while the Raman response causes an in- 
crease in the refractive index whose peak is shifted from the peak of pulse. In both illustrations, the value of the effective (average) refractive index, defined by

$$
\Delta n_{K}^{\mathrm{eff}}=\frac{1}{T} \int_{-\infty}^{\infty} \Delta n_{K}(t) d t,
$$

are taken to be the same, such that different effects of delayed nonlinearity on the dynamics of propagation can be well investigated. Moreover, in experiments one usually deals with the effective nonlinear refractive index, rather than the individual contributions.

Note that $\Delta n_{K}^{\text {eff }}$ is a function of pulse duration $T$ and delay time $\tau$. It is seen in Fig. 1 that the presence of the delayed Kerr reduces the nonlinearity in the front of the pulse and counteracts the negative plasma-induced nonlinearity generated at the back of the pulse. We performed a series of simulations with various values of $\left(g, g^{*}\right)$ subject to the previously mentioned condition that for a given initial pulse form, the calculated refractive index changes because instantaneous and retarded Kerr nonlinearity are the same. For the purpose of presentation here, we choose the value of $\left(g, g^{*}\right)$ to be $(1,0),(0.5,0.5),(0.25,0.75)$, and $(0,1)$. The pulse duration was set to be $60 \mathrm{fs}$ (at FWHM). Simulations were also performed for $\left(g, g^{*}\right)=(0.75,0.25)$, but we do not show the results since it was found that the dynamics does not change significantly from that of $\left(g, g^{*}\right)=(1,0)$. The laser energy was $2 \mathrm{~mJ}$, which corresponds with a peak power $33 \mathrm{GW}$, i.e., about 10 times larger than the critical power $3.3 \mathrm{GW}$. The laser wavelength was centered at $789 \mathrm{~nm}$. The initial collimated beam radius was $0.5 \mathrm{~mm}$.

\section{A. Filamentary laser pulses for various fractions of delayed Kerr nonlinearity}

In Fig. 2, the averaged intensities, obtained from simulations using four different pairs of $\left(g, g^{*}\right)$, chosen to be $(1,0)$, $(0.5,0.5),(0.25,75)$, and $(0,1)$ are shown, respectively, from top to bottom. Here, the averaged intensity is defined as

$$
I_{\mathrm{av}}(r, z)=\frac{1}{T} \int_{-\infty}^{\infty} I(r, z, t) d t .
$$

The use of this quantity is justified since it is impossible to follow the instantaneous intensity if the whole pulse duration is less than 100 femtosecond.

It is seen that the filaments exist for all cases. The radius of the filament is smaller when the contribution of instantaneous part becomes higher, which is an indication that the filament is more tightly bounded due to stronger selffocusing. Furthermore, in general the appearance of the filaments is quite different in two intervals, i.e., an interval near the nonlinear focal point (the short distance filament) and that in long distance after the nonlinear focal point. The filaments near the focal point are more structured than the latter ones. It is also clear that a higher contribution of delayed Kerr nonlinearity leads to a more stable filament.

The filamentation at the initial stage of propagation can be attributed to the moving focus effect [7]. According to this model, in the presence of self-focusing, the focal point
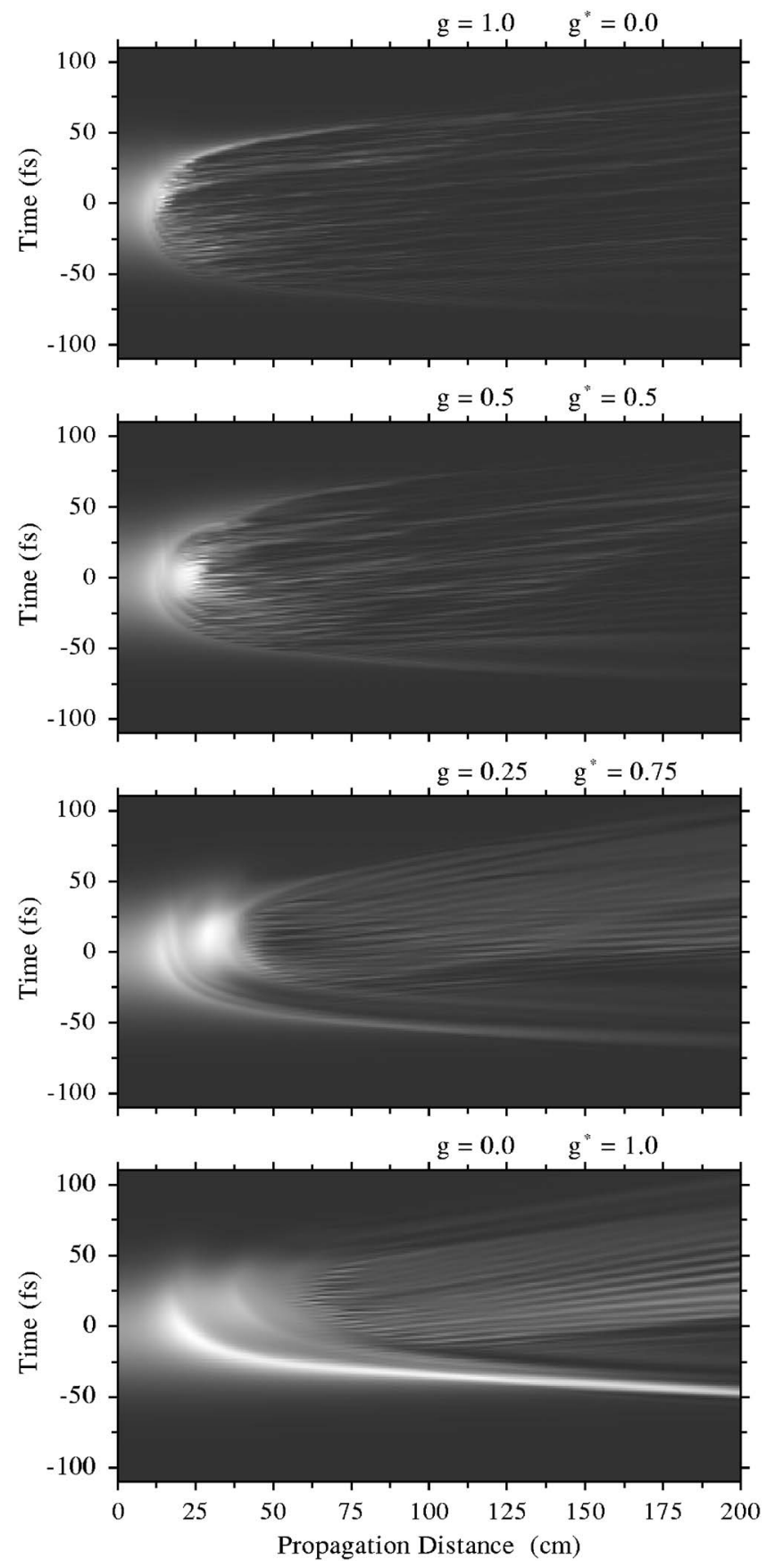

FIG. 4. The temporal dynamics of filaments inside circular area of radii $0.2 \mathrm{~mm}$ for various pairs of $g$ and $g^{*}$.

moves forth and back along the propagation axis and the focal point can be determined using relation $z_{f R}=\left(1 / z_{f}\right.$ $+1 / f)^{-1}$, where

$$
z_{f} \approx \frac{0.367 k_{0} r_{0}^{2}}{\left(\left\{\left[P(t) / P_{\mathrm{cr}}\right]^{1 / 2}-0.852\right\}^{2}-0.0219\right)^{1 / 2}}
$$

is the nonlinear focal length. Here, $r_{0}$ is the beam radius, $P(t)$ and $P_{\mathrm{cr}}$ are the power at the respective time slices and the critical power, respectively. In case that the beam is collimated, the linear focal length is $f=\infty$ such that $z_{f}=z_{f R}$. 

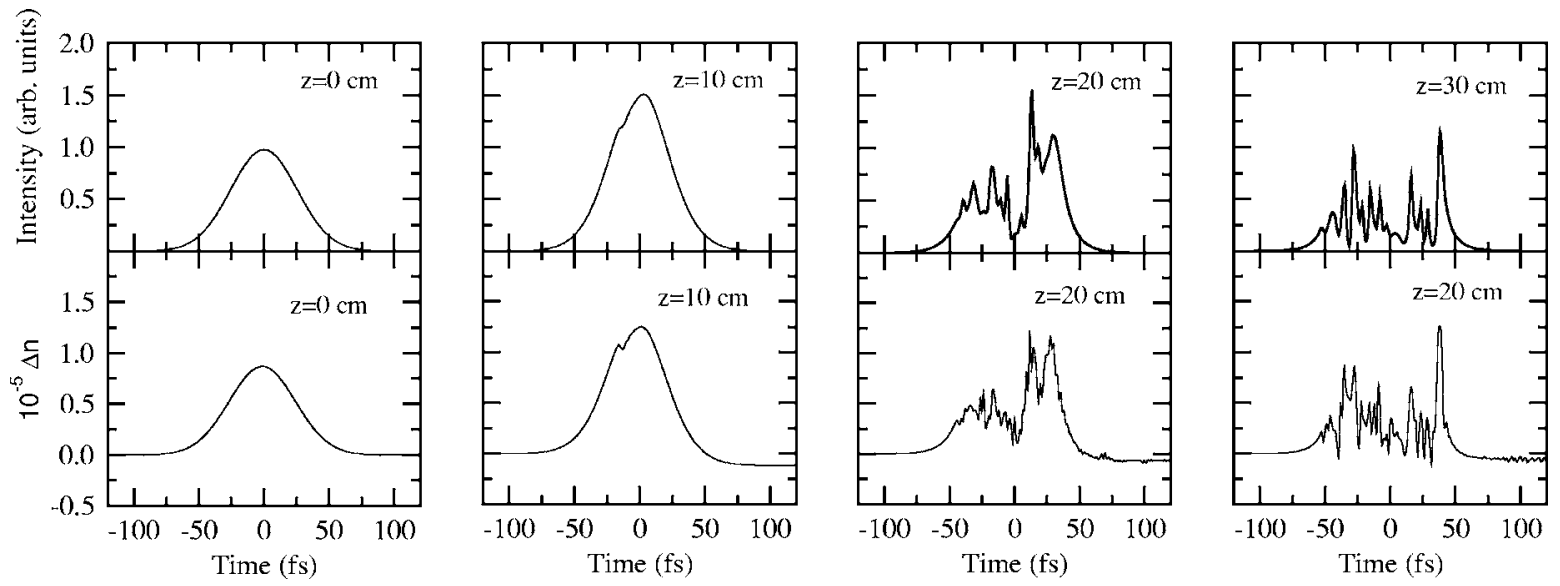

FIG. 5. The temporal profile of intensities (top) and refractive index change (bottom) obtained at a few tens cm of propagation distance. This plot results from a simulation using purely instantaneous Kerr nonlinearity.

It is clear from Eq. (13) that the nonlinear focal length depends on the power; this dependence in different time slices results into an extended interval of propagation. Moreover, in the case of a purely instantaneous medium $g=1$, the critical power $P_{\text {cr }}$ in the front of the pulse is smaller than when delayed Kerr nonlinearity is present. This results into smaller values of $z_{f}$ as seen in Fig. 2. We observed that in the case of $\left(g, g^{*}\right)=(1,0)$, the self-focusing occurs at $z_{f}$ $=9.5 \mathrm{~cm}$, while for $(0.5,0.5),(0.25,0.75)$, and $(0,1)$, we obtained $z_{f}$ to be $12 \mathrm{~cm}, 14 \mathrm{~cm}$, and $18 \mathrm{~cm}$, respectively.

In the next stage of propagation, the dynamics is much more complex since it involves nonlinear Kerr self-focusing, pulse splitting, and self-phase modulation modified by ionization. In case of a medium with a small fraction of delayed Kerr effect (or purely instantaneous), transverse structures are immediately apparent after self-focusing, but these gradually disappear or are delayed when the delay effect becomes relatively larger. Also, the intensity of the filaments exhibits oscillations and the shortest and most irregular os- cillation periods are found without delay, in case $\left(g, g^{*}\right)$ $=(1,0)$. In addition, there are traces of early beam diffraction, at $z$ around $25 \mathrm{~cm}$ for $\left(g, g^{*}\right)=(1,0)$, at $30 \mathrm{~cm}$ for $\left(g, g^{*}\right)=(0.5,0.5)$ and at $z \approx 40 \mathrm{~cm}$ for $(0.25,0.75)$.

Despite different values for $\left(g, g^{*}\right)$, the whole mechanism of the filamentation stage is generic. The beam in the frontier part of the pulse self-focuses and generates a plasma, and the resulting negative plasma nonlinearity suppresses the beam in the trailing edge. The plasma refractive index then changes strongly and depletes the beam in the central area; because of higher nonlinearity in the peripheral area, selffocusing occurs resulting in the rotational symmetric case that we consider here, into the formation of a ring. The interplay between suppression and self-focusing along the transversal coordinate then leads to the formation of multiple transversal rings. The number of rings, following the simulation results in fused silica [29], have been shown to depend on the input power of the pulse, i.e., the higher the input power, the more number of transverse rings. Recently, the
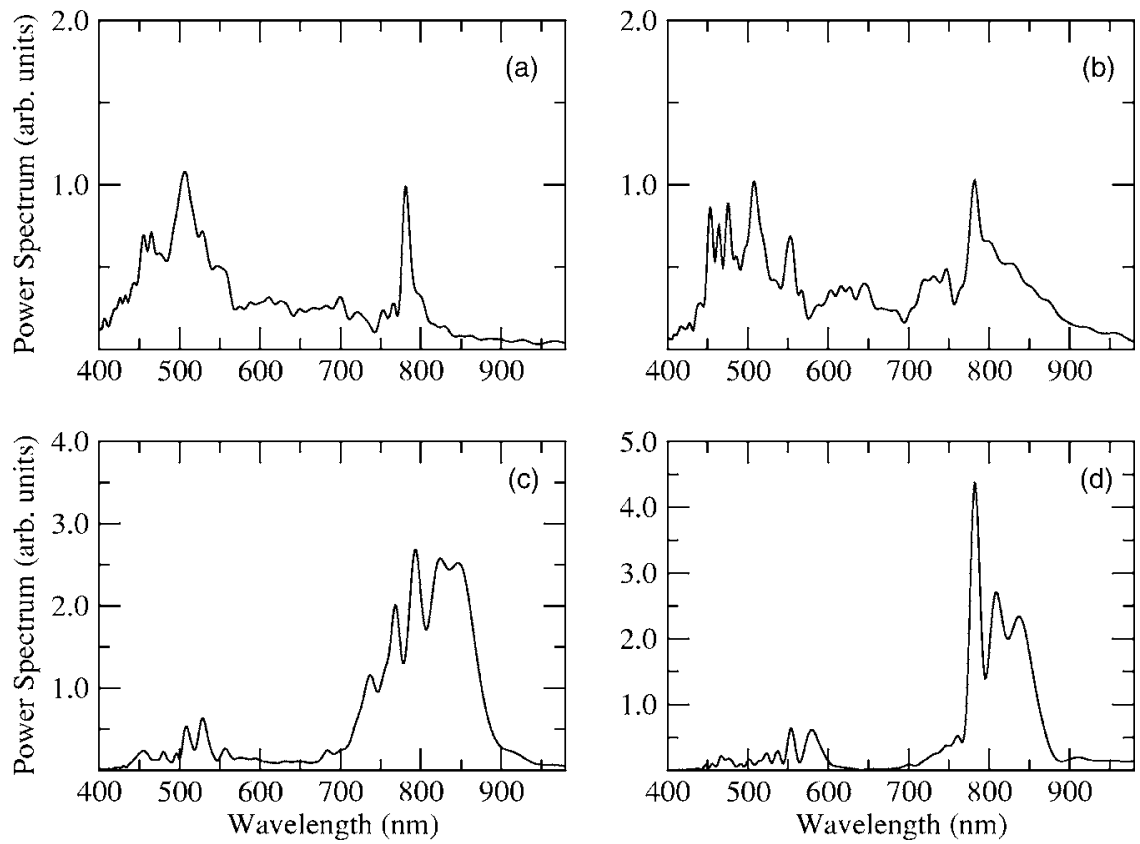

FIG. 6. The spectral power laser pulses at the end of the propagation distance for various pairs $\left(g, g^{*}\right), \quad$ i.e., $\quad(1$ $0),(0.5,05),(0.25,0.75)$, and $(0,1)$, respectively, for $(\mathrm{a}),(\mathrm{b}),(\mathrm{c})$, and (d). 
transverse ring formation has been observed experimentally in air using $85 \mathrm{~mJ}, 350 \mathrm{fs}$ laser pulses [26].

The next step in the propagation shows that the power in the leading edge decreases due to depletion by the MPI and the plasma formation stops, while the beam in the trailing edge regains power from the peripheral area, and refocusing occurs. This process repeatedly takes place until the power in each spitted pulse decreases below the limit for self-focusing or a quasibalance condition between self-focusing, plasma defocusing, and diffraction is created. When the power confined in the leading split pulses depletes rapidly due to multiphoton absorption such that the power required to clamp the filament down below the critical power, the beam in the respective time slices diffracts and one observes the track of early diffraction. Since the strength of diffraction depends inversely on the radius of the filament, the early diffraction is more obvious in case of $\left(g, g^{*}\right)=(1,0)$.

In Figs. 3(a)-3(d), the total energies of the pulse are shown as a function of propagation distance for four different values of $\left(g, g^{*}\right)$. In the inset of each figure, the pulse energy trapped in the leading part is plotted as lines, while that in the trailing part is displayed using the dotted curve. Here the leading part is defined as pulse components queuing before $T / 2$, with $T$ being the total pulse duration, and the trailing parts are those after $T / 2$. It can be seen that in case of $\left(g, g^{*}\right)=(1,0)$ and $\left(g, g^{*}\right)=(0.5,0.5)$, energy depletion mostly takes place in the leading part. These figures, together with those shown in Fig. 3 and in Fig. 2, support the mechanism of the early beam diffraction observed in the initial stage of propagation. In addition, the power transfer at propagation distances after the nonlinear focal point from the leading to the trailing edge as appears in case of $\left(g, g^{*}\right)$ $=(1,0)$ and $\left(g, g^{*}\right)=(0.5,0.5)$ can be attributed as an effect of dispersion due to different group velocity of each split pulse, as will be shown shortly in the next section. Also, most of the energy depletion takes place in the region near the nonlinear focal point, where the dynamics and competition between self-focusing, defocusing, and diffraction are most pronounced.

After long propagation distances, the total energy is almost constant, indicating that each split pulse propagates in a quasibalanced way, as mentioned above. We also noticed that the energy inside the filament after propagating $2 \mathrm{~m}$ was $4 \%$ for purely instantaneous Kerr effect and increased to $18 \%$ in the case of purely delayed Kerr effect.

\section{B. The mechanism of pulse splitting}

In order to better understand the origin of the irregular oscillations appearing in the intensity profile of the filaments, in Fig. 4 we display the temporal profile of the intensity. This intensity profile was obtained by a spatial averaging of the time dependent intensity over the circular area with radius of $0.2 \mathrm{~mm}$.

It is seen that there are two mechanism of pulse splitting, one which is due to refocusing of the trailing edge and the other due to a limitation of the intensity by MPI or tunneling ionization. The former mechanism takes place near the nonlinear focal point, owing to competition between the parent pulse and the generated pulses by the refocusing processes, while the latter applies mostly after limitation of intensity growth by the MPI or tunneling ionization.

In the case of $\left(g, g^{*}\right)=(1,0)$ (top figure), immediately after self-focusing, the pulse splits repeatedly and forms a comblike structure. Because of the instantaneous responses, the beam in all pulse slices undergoes simultaneous selffocusing which is then immediately followed by plasma generation. The generated plasma suppresses the intensity just in the back of the pulse and causes a comblike structure. The refocusing is less evident in this case, because of the rapid occurrence of simultaneous multiple splitting in all pulse components. As the split pulses propagate forward, the superposition of them creates an image of which the peak intensity oscillates.

In the presence of delayed Kerr nonlinearity, on the contrary, self-focusing first occurs in the trailing edge of the pulse, because of the greater nonlinearity in the respective time slices. The trace of pulse steepening can be seen, e.g., the temporal position of self-focusing, which always moves to the front part of the pulses. The higher intensity in the front part generates plasma and suppress the trailing part. As the beam in the backward part of the pulse regains power from the peripheral area, refocusing takes place and generates a pulse in the trailing part. By carefully inspecting Figs. 2 and 4 it is clear that oscillations in the peak intensity shown in Fig. 2 originate from the refocusing processes of the beam in the trailing part.

Next, as the intensity increases, the MPI limits the growth and the pulse undergoes multiple splitting. From the figures it is clear that the propagation of the split pulse is stable, owing to the quasibalance between self-focusing, diffraction, and plasma induced nonlinearity. We observed the width of split pulse is around $6 \mathrm{fs}$, in good agreement with the analytical results [16]. Also, the distance between the split pulses becomes wider owing to the different group velocity dispersion of the split pulses.

In Fig. 5, for the case of purely instantaneous Kerr nonlinearity, the temporal fluctuation of the intensity (top) and the corresponding time dependent nonlinear index changes (bottom), are displayed. Averaging is done over a circular area of radius $0.2 \mathrm{~mm}$. One can see that the temporal peaks strongly correlate with the maximum of the refractive index change, while the suppression directly links with the plasma dip. From this it is now clear that the origin of comblike structures shown in Fig. 4 originates from dynamic modulation instabilities because of the immediate response of selffocusing by the generation of plasma.

In Fig. 6, the power spectra are shown. By comparing the spectrum generated in the case of pure instantaneous nonlinearity with that in the presence of delayed nonlinearity, one may deduce that the effect of the delayed Kerr nonlinearity shows itself in a spectral shift from shorter to longer wave components. This conclusion is qualitatively in agreement with experimental evidences [10] and simulation results [20]. This shift is in contradiction with that resulting from plasma dispersion, since in a plasma the spectrum peak moves from longer to shorter wave components. Thus, the blueshifting effect observed in the case of $\left(g, g^{*}\right)=(1,0)$ and $(0.5,0.5)$ originates from plasma dispersion, while the bunches appear- 


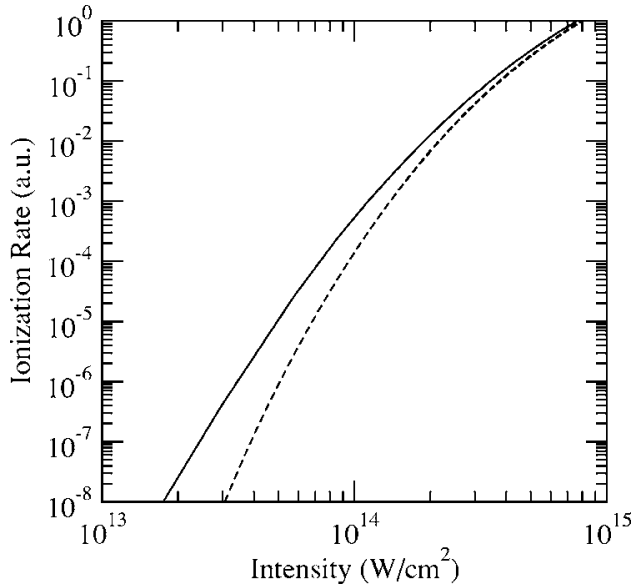

FIG. 7. The ionization rate of air calculated using PPT (solid curve) and ADK formula (dotted curve). The air molecules are assumed to be composed from $80 \%$ of $\mathrm{N}_{2}$ and $20 \%$ of $\mathrm{O}_{2}$ molecules.

ing in the short wavelength components, visible in the simulations for $\left(g, g^{*}\right)=(0.25,0.75)$ and $(0,1.0)$ are associated with the small fraction of pulses that remains in the filament, and thus strongly under the influence of the plasma.

\section{The stability of filament on the ionization rate}

The stability of the filament formed by propagation of intense, femtosecond laser pulses in air is not only determined by the Kerr nonlinearity (instantaneous and delayed), but also by the onset of ionization through a pseudobalance between self-focusing induced by the Kerr nonlinearity and plasma defocusing and diffraction. Because the proportional factor of plasma density to the plasma-induced refractive index is large [see Eq. (6)], a small deviation of ionization rate or plasma density from the correct value could strongly change the dynamics of the filament.

To demonstrate how a small deviation in the ionization rate could strongly modify the dynamics of the filament, we carried out simulations with ionization rate generated by ADK [25] and the PPT [31] formula. As it has been shown in Ref. [26] that by using an appropriate effective nuclear charge $Z_{\text {eff }}$, i.e., $Z_{\text {eff }}=0.9$ for $\mathrm{N}_{2}$ and $Z_{\text {eff }}=0.53$ for $\mathrm{O}_{2}$ mol- ecules, the PPT formula could reproduce the correct ionization rate. On the other hand, using the same $Z_{\text {eff }}$, the ionization rate generated by ADK formulas is in general smaller, particularly for intensity less than $2 \times 10^{14} \mathrm{~W} / \mathrm{cm}^{2}$. For the sake of comparison, in Fig. 7 the ionization rate of air molecules, assumed to be composed from $80 \%$ of $\mathrm{N}_{2}$ and $20 \%$ of $\mathrm{O}_{2}$, are compared. The solid curve stands for ionization rate calculated using PPT and the dashed curve for that obtained using ADK formulas. One can see that the discrepancies in the ionization rate become larger at lower intensity (by a factor greater than 5 for $I<10^{14} \mathrm{~W} / \mathrm{cm}^{2}$ ), but tends to coincide with each other at very high intensity $(>5$ $\times 10^{15} \mathrm{~W} / \mathrm{cm}^{2}$ ).

In Fig. 8 two simulated filaments are depicted; the top panel for result with ionization rate derived from PPT formula, while the bottom panel for that obtained using ADK formula. In both simulations, the value of $g$ and $g^{*}$ were set to be $(0.4,0.6)$. It is clear from Fig. 8 that the use of ADK formulas would underestimate the stability of the filament formation.

The reason for more stable filament can be explained as follows. For a given intensity, the PPT formula could provide higher ionization rate than that of ADK formula, such that the yield of the time dependent plasma density becomes larger. Since plasma defocusing proportionally depends on the plasma density, the balance between Kerr self-focusing, plasma defocusing, and diffraction can be fulfilled at relatively low intensity. Consequently, the beam is less bounded, because of lower intensity and thus lower self-focusing. This also explains why the filament's diameter is also found to be larger.

\section{CONCLUSIONS}

We have investigated through numerical simulations of the extended nonlinear Schrödinger equation the effect of different contributions of the delayed Kerr nonlinearity on the stability of filamentary pulses in air. It is found that the pulse will be much more stable while propagating in the medium when the relative fraction of delayed nonlinearity is larger. We argued that the reason for this is that the presence of delayed nonlinearity declines the self-focusing effect in
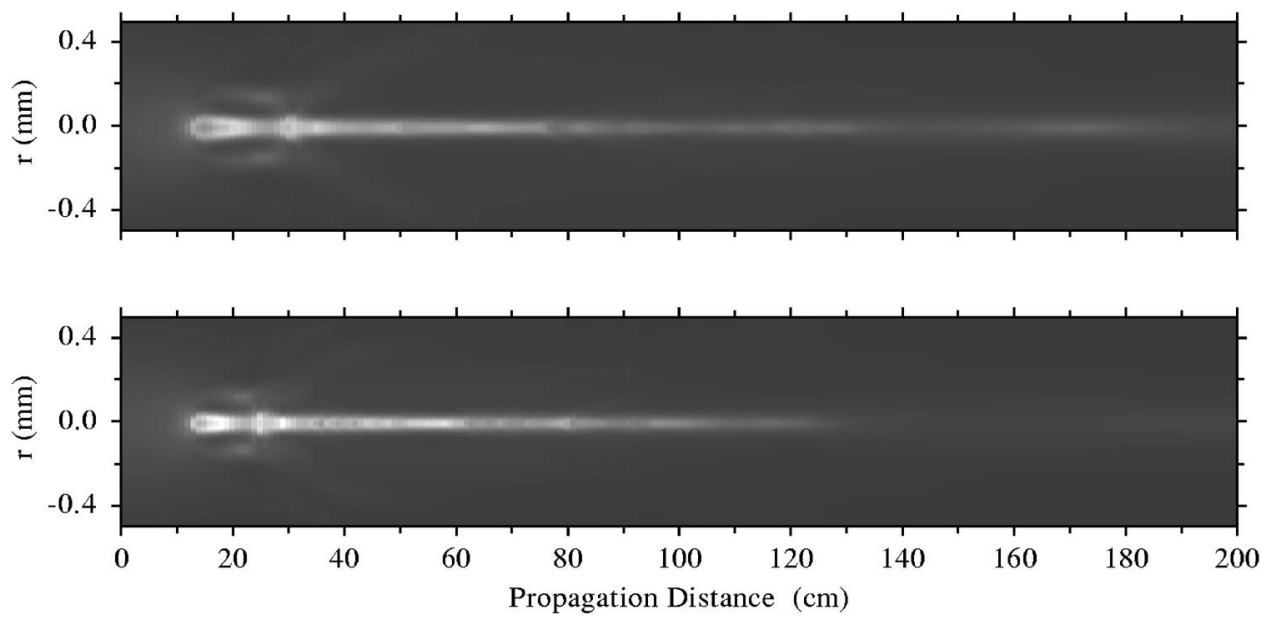

FIG. 8. Comparison of simulated filaments with ionization rate generated using PPT (top panel) and ADK formula (bottom). 
front of the pulse and counteracts the plasma generated nonlinearity at the trailing edges.

It has also been found that the filamentation is a generic process. The mechanism of filamentation near the nonlinear focal point is mainly driven by the dynamic modulation instability resulting from competition between Kerr selffocusing, diffraction and plasma defocusing, while at longer distance this is due to pseudobalance between Kerr selffocusing, diffraction and plasma defocusing.

The pulse splitting that is observed is based on two mechanisms; the first is the competition between the leading pulse and that generated by refocusing of the trailing part, and the second is the limitation of the growth in the intensity by MPI or tunneling ionization. The apparent effect of the delayed nonlinearity is shown also in a spectral shift from shorter to longer wave components.
Filament's stability with respect to ionization rate is also investigated. Two formulas, i.e., the PPT and ADK, are used to generate the ionization rate. It is found that larger ionization rate could yield more stable and longer filament, due to a pseudobalance mechanism between self-focusing and plasma defocusing and diffraction, which can be fulfilled at relatively low intensity.

\section{ACKNOWLEDGMENTS}

The authors acknowledge financial contribution from the Koninklijke Nederlandse Akademie van Wetenschappen (KNAW) from the Mobility Programme Project 03-MP-15 within the Scientific Programme Indonesia-Netherlands, which accommodated a visit of M.N. to the University of Twente, Enschede, the Netherlands, and a visit of E.vG. to Indonesia.
[1] M. D. Perry and G. Mourou, Science 264, 917 (1994).

[2] H. Wille, M. Rodriguez, J. Kasparian, D. Mondelain, J. Yu, A. Mysyrowicz, R. Sauberry, J. P. Wolf, and L. Wöste, Eur. Phys. J.: Appl. Phys. 20, 183 (2002).

[3] E. T. J. Nibbering, P. F. Curley, G. Grillon, B. S. Prade, M. A. Franco, F. Salin, and A. Mysyrowicz, Opt. Lett. 21, 62 (1996); Q. Feng, J. V. Moloney, A. C. Newell, E. M. Wright, K. Cook, P. K. Kennedy, D. X. Hammer, and R. C. Thompson, IEEE J. Quantum Electron. 33, 127 (1997).

[4] G. T. Boyd, J. Opt. Soc. Am. B 6, 685 (1989); J. W. Wu, J. R. Heffin, R. A. Norwood, K. Y. Wong, O. Zamani-Khamiri, A. F. Garito, P. Kalyanaraman, and J. Sounik, ibid. 6, 707 (1990).

[5] L. Wöste et al., Laser Optoelektron. 29, 51 (1997); H. Schillinger and R. Sauerbrey, Appl. Phys. B: Lasers Opt. 68, 753 (1999).

[6] A. Braun, G. Korn, X. Liu, D. Du, J. Squier, and G. Mourou, Opt. Lett. 20, 73 (1995).

[7] A. Brodeur, C. Y. Chien, F. A. Ilkov, S. L. Chin, O. G. Kosareva, and V. P. Kandidov, Opt. Lett. 22, 304 (1997).

[8] E. T. J. Nibbering, P. F. Curley, G. Grillon, B. S. Prade, M. A. Franco, F. Salin, and A. Mysyrowicz, Opt. Lett. 21, 62 (1996).

[9] M. Mlejnek, E. M. Wright, and J. V. Moloney, Opt. Lett. 23, 382 (1998).

[10] E. T. J. Nibbering, G. Grillon, M. A. Franco, B. S. Prade, and A. Mysyrowicz, J. Opt. Soc. Am. B 14, 650 (1997).

[11] M. Rodriguez et al., Phys. Rev. E 69, 036607 (2004); J. Kasparian et al., Science 301, 61 (2003).

[12] N. Aközbek, M. Scalora, C. M. Bowden, and S. L. Chin, Opt. Commun. 191, 353 (2001).

[13] M. Nurhuda, A. Suda, M. Hatayama, K. Nagasaka, and K. Midorikawa, Phys. Rev. A 66, 023811 (2002).

[14] S. Tzortzakis, L. Bergé, A. Couairon, M. Franco, B. Prade, and A. Mysyrowics, Phys. Rev. Lett. 86, 5470 (2001).

[15] L. Bergé and A. Couairon, Phys. Plasmas 7, 210 (2000).
[16] P. Sprangle, J. R. Penano, and B. Hafizi, Phys. Rev. E 66, 046418 (2002).

[17] T. Brabec and F. Krausz, Phys. Rev. Lett. 78, 3282 (1997).

[18] K. Burnet, V. C. Reed, and P. L. Knight, J. Phys. B 26, 561 (1993).

[19] A. Couairon and L. Bérge, Phys. Plasmas 7, 193 (2000).

[20] S. L. Chin, N. Aközbek, A. Proulx, S. Petit, and C. W. Bowden, Opt. Commun. 188, 181 (2001).

[21] A. Couairon, S. Tzortzakis, L. Berge, M. Franco, B. Prade, and A. Mysyrowicz, J. Opt. Soc. Am. B 19, 1117 (2002).

[22] E. T. J. Nibbering, G. Grillon, M. A. Franco, B. S. Prade, and A. Mysyrowicz, J. Opt. Soc. Am. B 14, 650 (1997).

[23] J. F. Ripoche, G. Grillon, B. Prade, M. Franco, E. Nibbering, R. Lange, and A. Mysyrowicz, Opt. Commun. 35, 310 (1997).

[24] L. V. Keldysh, Zh. Eksp. Teor. Fiz. 47, 1945 (1964) [Sov. Phys. JETP 20, 1307 (1965)].

[25] M. V. Ammosov, N. B. Delone, and V. P. Krainov, Zh. Eksp. Teor. Fiz. 91, 2008 (1986) [Sov. Phys. JETP 64, 1191 (1987)].

[26] A. Talebpour, J. Yang, and. S. L. Chin, Opt. Commun. 163, 29 (1999).

[27] I. S. Golubtsov, V. P. Kandidov, and O. G. Kosareva, Quantum Electron. 33, 525 (2003).

[28] C. Courtois, A. Couairon, B. Cros, J. R. Marques, and G. Matthieussent, Phys. Plasmas 8, 3445 (2001).

[29] K. Ishikawa, H. Kumagai, and K. Midorikawa, Phys. Rev. E 66, 056608 (2002).

[30] M. Mlejnek, E. M. Wright, and J. V. Moloney, Opt. Express 4, 223 (1999).

[31] A. M. Perelemov, V. S. Popov, and M. V. Terent'ev, Zh. Eksp. Teor. Fiz. 50, 1393 (1966), [Sov. Phys. JETP 23, 924 (1966)].

[32] M. Nurhuda, A. Suda, and K. Midorikawa, Phys. Rev. A 66, 041802 (2002).

[33] J. K. Ranka and A. L. Gaeta, Opt. Lett. 23, 534 (1998). 